\title{
Density perturbations in a brane-world universe with dark radiation
}

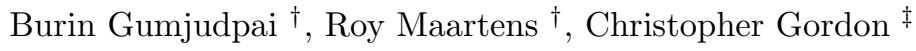 \\ ${ }^{\dagger}$ Institute of Cosmology and Gravitation, University of Portsmouth, Portsmouth PO1 2EG, UK \\ ${ }^{\ddagger}$ DAMTP, Centre for Mathematical Sciences, University of Cambridge, Cambridge CB3 OWA, UK
}

\begin{abstract}
We investigate the effects on cosmological density perturbations of dark radiation in a RandallSundrum 2 type brane-world. Dark radiation in the background is limited by observational constraints to be a small fraction of the radiation energy density, but it has an interesting qualitative effect in the radiation era. On large scales, it serves to slightly suppress the radiation density perturbations at late times, while boosting the perturbations in dark radiation. In a kinetic (stiff) era, the suppression is much stronger, and drives the density perturbations to zero.
\end{abstract}

\section{INTRODUCTION}

In brane-world cosmology, which has emerged in the context of recent developments in $\mathrm{M}$ theory, the observable universe is a $1+3$-dimensional "brane" surface embedded in a $1+3+d$-dimensional "bulk" spacetime. Fields and particles in the non-gravitational sector are confined to the brane, while gravity propagates in the bulk. Simple phenomenological brane-world models are the cosmological generalizations [1-4] of the Randall-Sundrum 2 model [5], which has a self-gravitating brane with Minkowski geometry embedded in an infinite part of 5-dimensional Anti de Sitter space $\left(\mathrm{AdS}_{5}\right)$. Gravity on the brane is prevented from "leaking" into the infinite extra dimension at low energies via "warping" of the metric by the negative bulk cosmological constant, $\Lambda_{5}=-6 / \ell^{2}$, where $\ell$ is the curvature radius of $\mathrm{AdS}_{5}$. Modes of the 5D graviton have an effective mass on the brane, since the projection onto the brane of a null $5 \mathrm{D}$ graviton momentum vector is in general a $4 \mathrm{D}$ timelike momentum vector. The massless mode arises when the projection is null, and corresponds to the $4 \mathrm{D}$ graviton, which is dominant at low energies. The massive Kaluza-Klein (KK) modes produce corrections to the gravitational potential in the weak-field static limit, which are $O\left(\ell^{2} / r^{2}\right)[5,6]$. Table-top experiments currently impose an upper bound $\ell \lesssim 0.1 \mathrm{~mm}$.

On the brane, the negative $\Lambda_{5}$ is offset by the positive brane tension $\lambda$, and the effective cosmological constant on the brane is

$$
\Lambda=\frac{1}{2}\left(\Lambda_{5}+\kappa^{2} \lambda\right)
$$

where $\kappa^{2}=8 \pi / M_{4}^{2}$ and $M_{4} \sim 10^{19} \mathrm{GeV}$ is the effective Planck scale on the brane. Fine-tuning can set $\Lambda=0$. Because of the large extra dimension, the true fundamental gravity scale can be as low as $\sim \mathrm{TeV}$ in some brane-world scenarios, but in generalized RS2 models it is higher:

$$
M_{5}^{3}=\frac{M_{4}^{2}}{\ell}, \ell<0.1 \mathrm{~mm} \Rightarrow M_{5}>10^{5} \mathrm{TeV}, \lambda>(100 \mathrm{GeV})^{4} .
$$

At high energies $(\rho \gg \lambda)$ in the early universe, gravity becomes 5-dimensional and there are significant corrections to standard cosmological dynamics.

The unperturbed cosmological brane-world is a Friedmann brane in a Schwarzschild-AdS 5 bulk [2] (where the expansion of the universe can be interpreted as motion of the brane in the bulk), satisfying the usual energy conservation equation but modified Friedmann equations. For a spatially flat universe without cosmological constant, these are

$$
\begin{aligned}
H^{2} & =\frac{\kappa^{2}}{3} \rho\left(1+\frac{\rho}{2 \lambda}\right)+\frac{m}{a^{4}}, \\
\dot{H} & =-\frac{\kappa^{2}}{2} \rho(1+w)\left(1+\frac{\rho}{\lambda}\right)-2 \frac{m}{a^{4}},
\end{aligned}
$$

where $w=p / \rho$ and $m$ is a constant, proportional to the mass of the bulk black hole. To avoid a naked singularity in the bulk, we take $m \geq 0$. The tidal, Coulomb effect of the 5-dimensional black hole on the brane is an effective radiative $\left(\propto a^{-4}\right)$ term, the so-called "dark radiation". Nucleosynthesis is sensitive to the inclusion of additional relativistic energies not thermally coupled to the radiation plasma, and so is the cosmic microwave background. This places limits on the amount of dark radiation compatible with observations [7-9] 


$$
\left(\frac{\rho^{*}}{\rho_{\text {rad }}}\right)_{\text {nuc }} \lesssim 0.03
$$

so that $\rho^{*} \ll \rho_{\text {rad }}$ always, and the dark radiation is negligible by the matter era. The quadratic correction terms $\rho^{2} / \lambda$ are dominant at high energies, $\rho \gg \lambda$, and negligible at low energies. Since $\lambda \gg 1 \mathrm{MeV}$, the quadratic effects are negligible by nucleosynthesis. At late times, the standard 4-dimensional evolution is therefore recovered.

In the absence of dark radiation, the exact solution (with $\dot{w}=0=m$ ) is [1]

$$
a=\operatorname{const}\left[t\left(t+t_{\lambda}\right)\right]^{1 / 3(w+1)}, \quad t_{\lambda}=\frac{M_{4}}{\sqrt{\pi \lambda}}<10^{-9} \mathrm{sec} .
$$

In the radiation era, $w=\frac{1}{3}$, the exact solution with dark radiation $(m \neq 0)$ is [7]

$$
a=\operatorname{const}\left[t\left(t+t_{\lambda}\right)\right]^{1 / 4}, \quad t_{\lambda}=\frac{\sqrt{3} M_{4}}{4 \sqrt{\pi \lambda}\left(1+3 m / \kappa^{2} \rho a^{4}\right)} .
$$

At late times, $t \gg t_{\lambda}$, we recover the standard solution $a \propto t^{2 / 3(w+1)}$, whereas at early times (high energies), $t \ll t_{\lambda}$, the evolution is very different: $a \propto t^{1 / 3(w+1)}$.

The high-energy brane-world correction provides increased Hubble damping in inflation; instead of $H \propto \sqrt{V}$ as in general relativity, the modified Friedmann equation (3) shows that $H \propto V$ when $V \gg \lambda$. This means in particular that slow-roll inflation is possible even for potentials $V(\varphi)$ that would be too steep in standard cosmology $[4,10]$. In addition, large-scale scalar perturbations generated by high-energy slow-roll inflation have an enhanced amplitude $A_{\mathrm{s}}$ compared with the standard general relativity amplitude $\left(A_{\mathrm{s}}\right)_{\mathrm{gr}}[4]$ :

$$
A_{\mathrm{s}}^{2} \approx\left(A_{\mathrm{s}}^{2}\right)_{\mathrm{gr}}\left(\frac{V}{\lambda}\right)^{2}
$$

The background dynamics of brane-world cosmology are simple because they are effectively 4-dimensional. Cosmological perturbations introduce truly 5-dimensional degrees of freedom, so that the 5 -dimensional bulk perturbation equations must be solved in order to solve for perturbations on the brane. These 5-dimensional equations are partial differential equations for the 3-dimensional Fourier modes, subject to complicated initial and boundary conditions. However, on large scales, if one can neglect gradient terms, the density or curvature perturbations can be determined without knowing the 5-dimensional solutions $[4,11,8]$. The qualitative 5-dimensional effects on large-scale density perturbations during inflation and the transition to radiation-domination were investigated via a toy model in [12]. Here we generalise the results of [12] to include the dark radiation term $m / a^{4}$ in the background. The presence of this term in the background can have a significant effect on the perturbations.

\section{FIELD EQUATIONS}

The effective field equations on the brane are derived from the $5 \mathrm{D}$ field equations in the bulk, ${ }^{(5)} G_{A B}=-\Lambda_{5}{ }^{(5)} g_{A B}$, by projecting the $5 \mathrm{D}$ curvature (using the Gauss-Codazzi equations), and then imposing the Darmois-Israel junction conditions at the brane (with $Z_{2}$-symmetry) [3]:

$$
G_{a b}=-\Lambda g_{a b}+\kappa^{2} T_{a b}+6 \frac{\kappa^{2}}{\lambda} \mathcal{S}_{a b}-\mathcal{E}_{a b} .
$$

Here $\mathcal{S}_{a b} \sim\left(T_{a b}\right)^{2}$ is the high-energy correction term, which is negligible for $\rho \ll \lambda$, while $\mathcal{E}_{a b}$ is the projection of the bulk Weyl tensor, encoding corrections from 5D graviton (KK) effects (and giving the dark radiation in the background). From the brane-observer viewpoint, the energy-momentum corrections in $\mathcal{S}_{a b}$ are local, whereas the KK corrections in $\mathcal{E}_{a b}$ are nonlocal $[11,13,14]$, since they incorporate 5D gravity wave modes. These nonlocal corrections cannot be determined purely from data on the brane, and so the effective field equations are not a closed system. One needs to supplement them by $5 \mathrm{D}$ equations governing $\mathcal{E}_{a b}$, which are obtained from the 5D Einstein and Bianchi equations [3].

The trace free $\mathcal{E}_{a b}$ contributes an effective KK energy density $\rho^{*}$, pressure $\rho^{*} / 3$, momentum density $q_{a}^{*}$ and anisotropic stress $\pi_{a b}^{*}$ on the brane,

$$
-\frac{1}{\kappa^{2}} \mathcal{E}_{a b}=\rho^{*}\left(u_{a} u_{b}+\frac{1}{3} h_{a b}\right)+q_{a}^{*} u_{b}+q_{b}^{*} u_{a}+\pi_{a b}^{*}
$$


where $u^{a}$ is a physically determined 4 -velocity on the brane and $h_{a b}=g_{a b}+u_{a} u_{b}$ projects into the comoving rest space at each event. The brane "feels" the bulk gravitational field through these terms. In the background, $q_{a}^{*}=0=\pi_{a b}^{*}$, since only the dark radiation term $\rho^{*}\left(\propto m a^{-4}\right)$ is compatible with Friedmann symmetry. The KK momentum density defines a velocity $v_{a}$ of the Weyl "fluid" relative to $u^{a}$, by $q_{a}^{*}=\rho^{*} v_{a}$.

For a perfect fluid or scalar field, we choose $u^{a}$ as the frame in which there is no energy flux. Then the effective total energy density and pressure are

$$
\begin{aligned}
\rho^{\mathrm{eff}} & =\rho\left(1+\frac{\rho}{2 \lambda}+\frac{\rho^{*}}{\rho}\right), \\
p^{\mathrm{eff}} & =\rho\left[w+(1+2 w) \frac{\rho}{2 \lambda}+\frac{\rho^{*}}{3 \rho}\right] .
\end{aligned}
$$

Energy-momentum conservation, $\nabla^{b} T_{a b}=0$, holds on the brane. Together with the $4 \mathrm{D}$ Bianchi identity, this implies that $\nabla^{a} \mathcal{E}_{a b}=6 \kappa^{2} \nabla^{a} \mathcal{S}_{a b} / \lambda$, which shows qualitatively how $1+3$ spacetime variations in the matter-radiation on the brane can source KK modes. These KK "conservation equations" in linearized form are

$$
\begin{aligned}
& \dot{\rho}^{*}+\frac{4}{3} \Theta \rho^{*}+\mathrm{D}^{a} q_{a}^{*}=0, \\
& \dot{q}_{a}^{*}+4 H q_{a}^{*}+\frac{1}{3} \mathrm{D}_{a} \rho^{*}+\frac{4}{3} \rho^{*} A_{a}+\mathrm{D}^{b} \pi_{a b}^{*}=-\frac{(\rho+p)}{\lambda} \mathrm{D}_{a} \rho,
\end{aligned}
$$

where $\Theta$ is the volume expansion rate ( $=3 H$ in the background), $A_{a}$ is the 4 -acceleration, and $\mathrm{D}_{a}$ is the covariant spatial derivative in the comoving rest space. Spatial inhomogeneity $\left(\mathrm{D}_{a} \rho \neq 0\right)$ is a source for KK modes. Qualitatively and geometrically this can be understood as follows [11,13]: the non-uniform 5D gravitational field generated by inhomogeneous 4D matter-radiation contributes to the 5D Weyl tensor, which nonlocally "backreacts" on the brane via its projection $\mathcal{E}_{a b}$. Equation (14) shows that the source term is suppressed at low energies, and during quasi-de Sitter inflation on the brane.

Equations (13) and (14) are propagation equations for $\rho^{*}$ and $q_{a}^{*}$. There is no propagation equation on the brane for $\pi_{a b}^{*}$, so that one cannot determine the KK modes purely from data on the brane. In the 1+3-covariant description of braneworld perturbations [11,15-17], the KK anisotropic stress $\pi_{a b}^{*}$ is isolated as the term that must be determined from $5 \mathrm{D}$ equations. Once $\pi_{a b}^{*}$ is determined in this way, the $1+3$ perturbation equations on the brane form a closed system. The KK terms act as source terms modifying the standard general relativity perturbation equations, together with the local high-energy corrections.

\section{DENSITY PERTURBATION EQUATIONS}

We define density and expansion (velocity) perturbation scalars, as in general relativity [18],

$$
\Delta=\frac{a^{2}}{\rho} \mathrm{D}^{2} \rho, Z=a^{2} \mathrm{D}^{2} \Theta
$$

and then define dimensionless KK perturbation scalars [11],

$$
U=\frac{a^{2}}{\rho} \mathrm{D}^{2} \rho^{*}, Q=\frac{a}{\rho} \mathrm{D}^{2} q^{*}, \Pi=\frac{1}{\rho} \mathrm{D}^{2} \pi^{*},
$$

where the scalar potentials $q^{*}$ and $\pi^{*}$ are defined by $q_{a}^{*}=\mathrm{D}_{a} q^{*}, \pi_{a b}^{*}=\left(\mathrm{D}_{a} \mathrm{D}_{b}-\frac{1}{3} h_{a b} \mathrm{D}^{2}\right) \pi^{*}$. The dark radiation fluctuation $U$ is present even if there is no dark radiation in the background $\left(\rho^{*}=0\right)$. It leads to an isocurvature (non-adiabatic) mode, even when the matter perturbations are assumed adiabatic [12]. We define the total effective dimensionless entropy $S^{\mathrm{eff}}$ via the non-adiabatic part of the effective pressure as [18]

$$
p^{\mathrm{eff}} S^{\mathrm{eff}}=a^{2} \mathrm{D}^{2} p^{\mathrm{eff}}-c_{\mathrm{eff}}^{2} a^{2} \mathrm{D}^{2} \rho^{\mathrm{eff}},
$$

where $c_{\text {eff }}^{2}=\dot{p}^{\text {eff }} / \dot{\rho}^{\text {eff }}$. Then

$$
S^{\mathrm{eff}}=\frac{\left[3 c_{\mathrm{s}}^{2}-1+\left(2+3 w+3 c_{\mathrm{s}}^{2}\right) \rho / \lambda\right]}{\left[(1+w)(1+\rho / \lambda)+4 \rho^{*} / 3 \rho\right]\left[3 w+3(1+2 w) \rho / 2 \lambda+\rho^{*} / \rho\right]}\left[\frac{4}{3} \frac{\rho^{*}}{\rho} \Delta-(1+w) U\right],
$$


where $c_{\mathrm{s}}^{2}=\dot{p} / \dot{\rho}$. If $\rho^{*}=0$ in the background, then $U$ is an isocurvature mode: $S^{\text {eff }} \propto U$. If $\rho^{*} \neq 0$ in the background, then the weighted difference between $U$ and $\Delta$ determines the isocurvature mode: $S^{\text {eff }} \propto\left(4 \rho^{*} / 3 \rho\right) \Delta-(1+w) U$. At very high energies, $\rho \gg \lambda$, the entropy is suppressed by the factor $\lambda / \rho$. At low energies in the radiation era $\left(c_{\mathrm{s}}^{2}=\frac{1}{3}\right)$ it is suppressed by the factor $\rho / \lambda$.

The covariant density perturbation equations on the brane [11] reduce to

$$
\begin{aligned}
& \dot{\Delta}=3 w H \Delta-(1+w) Z, \\
& \dot{Z}=-2 H Z-\left(\frac{c_{\mathrm{s}}^{2}}{1+w}\right) \mathrm{D}^{2} \Delta-\kappa^{2} \rho U-\frac{1}{2} \kappa^{2} \rho\left[1+(4+3 w) \frac{\rho}{\lambda}-\left(\frac{4 c_{\mathrm{s}}^{2}}{1+w}\right) \frac{\rho^{*}}{\rho}\right] \Delta, \\
& \dot{U}=(3 w-1) H U+\left(\frac{4 c_{\mathrm{s}}^{2}}{1+w}\right)\left(\frac{\rho^{*}}{\rho}\right) H \Delta-\left(\frac{4 \rho^{*}}{3 \rho}\right) Z-a \mathrm{D}^{2} Q, \\
& \dot{Q}=(3 w-1) H Q-\frac{1}{3 a} U-\frac{2}{3} a \mathrm{D}^{2} \Pi+\frac{1}{3 a}\left[\left(\frac{4 c_{\mathrm{s}}^{2}}{1+w}\right) \frac{\rho^{*}}{\rho}-3(1+w) \frac{\rho}{\lambda}\right] \Delta,
\end{aligned}
$$

where $\dot{f} \equiv u^{a} \partial_{a} f$, and we have corrected the minor errors in the equations given in [12]. The KK anisotropic stress term $\Pi$ occurs only via its Laplacian, $D^{2} \Pi$. If we can neglect this term on large scales, then the system of density perturbation equations closes on super-Hubble scales [11]. An equivalent statement applies to the large-scale curvature perturbations [8]. We note that in the low-energy approximation scheme used by Koyama [19],

$$
\Pi \propto \mathrm{D}^{2} U,
$$

so that in particular $\Pi \rightarrow 0$ on large scales.

On large scales in the braneworld, neglecting gradient terms, density perturbations are then described by the set $\{\Delta, Z, U, Q\}$, where $Q$ decouples from the other variables. KK effects introduce two new isocurvature modes on large scales, associated with $U$ and $Q[12,15]$.

We can find a first integral of the large-scale system by following the same approach used in general relativity [18]. A covariant local curvature perturbation is defined by the gradient of $R^{\perp}$, the 3 -Ricci scalar for $u^{a}$-observers. This scalar is given by the Codazzi equation on the brane [14],

$$
R^{\perp}=-\frac{2}{3} \Theta^{2}+2 \kappa^{2} \rho^{\mathrm{eff}} .
$$

Then the dimensionless curvature perturbation scalar is defined by $C=a^{4} \mathrm{D}^{2} R^{\perp}$. This gives

$$
C=-4 a^{2} H Z+2 \kappa^{2} a^{2} \rho\left(1+\frac{\rho}{\lambda}\right) \Delta+2 \kappa^{2} a^{2} \rho U .
$$

Using the large-scale equations (19)-(21), we find that $C$ is locally conserved (i.e., constant along fundamental worldlines) on large scales,

$$
C=C_{0}, \quad \dot{C}_{0}=0 .
$$

(The minor errors in [12] led to the erroneous conclusion that $C$ was not in general locally conserved on the brane.)

Thus $C$ leads to a first integral for the large-scale system. We also replace $\Delta$ by the dimensionless perturbation variable

$$
\Phi=\kappa^{2} a^{2} \rho \Delta .
$$

In general relativity, $\Phi$ is the covariant analogue of the Bardeen metric perturbation variable $\Phi_{H}$ [18]. In the braneworld, high-energy and KK effects mean that the analogue of $\Phi_{H}$ is a complicated generalisation of Eq. (27) (see [15]). The key problem in trying to use the generalised variable is that it contains $\Pi$, which remains undetermined. (This is the same reason that the large-scale Sachs-Wolfe effect on the brane cannot be determined from the density or curvature perturbations [8].) In any case, $\Phi$ as defined above leads to useful simplification of the perturbation equations.

The coupled system of Eqs. (19)-(21), which is closed on large scales (neglecting gradient terms), reduces via Eqs. (26) and (27) to the system

$$
\begin{aligned}
& \dot{\Phi}=-H\left[1+(1+w) \frac{\kappa^{2} \rho}{2 H^{2}}\left(1+\frac{\rho}{\lambda}\right)\right] \Phi-\left[(1+w) \frac{a^{2} \kappa^{4} \rho^{2}}{2 H}\right] U+\left[(1+w) \frac{\kappa^{2} \rho}{4 H}\right] C_{0}, \\
& \dot{U}=-H\left[1-3 w+\frac{2 \kappa^{2} \rho^{*}}{3 H^{2}}\right] U-\frac{2 \rho^{*}}{3 a^{2} H \rho}\left[1+\frac{\rho}{\lambda}-\frac{6 c_{\mathrm{s}}^{2} H^{2}}{(1+w) \kappa^{2} \rho}\right] \Phi+\left[\frac{\rho^{*}}{3 a^{2} H \rho}\right] C_{0} .
\end{aligned}
$$

This closed system for $\Phi, U$ generalises the equations given in [12] to the case $\rho^{*} \neq 0$. Once $\Phi$ and $U$ are solved for, $Q$ is directly determined by Eq. (22) with $\mathrm{D}^{2} \Pi$ set to zero. 


\section{SOLUTIONS}

The closed system for $\Phi$ and $U$ may be rewritten on using the Friedmann equation (3) and changing the time variable to the number of e-folds, $t \rightarrow N=\ln \left(a / a_{0}\right)$. This yields

$$
\begin{aligned}
\Phi^{\prime}=- & {\left[1+\frac{3(1+w)(1+\rho / \lambda)}{2\left(1+\rho / 2 \lambda+\rho^{*} / \rho\right)}\right] \Phi-\left[\frac{3(1+w)}{2\left(1+\rho / 2 \lambda+\rho^{*} / \rho\right)} a^{2} \kappa^{2} \rho\right] U+\left[\frac{3(1+w)}{4\left(1+\rho / 2 \lambda+\rho^{*} / \rho\right)}\right] C_{0}, } \\
U^{\prime}=- & {\left[1-3 w+\frac{2 \rho^{*} / \rho}{\left(1+\rho / 2 \lambda+\rho^{*} / \rho\right)}\right] U-\left[\frac{\rho^{*}}{\rho}\left\{\frac{(1+\rho / \lambda)}{\left(1+\rho / 2 \lambda+\rho^{*} / \rho\right)}-\frac{2 c_{\mathrm{s}}^{2}}{(1+w)}\right\} \frac{2}{a^{2} \kappa^{2} \rho}\right] \Phi } \\
& +\left[\frac{\rho^{*}}{\rho} \frac{1}{\left(1+\rho / 2 \lambda+\rho^{*} / \rho\right)} \frac{1}{a^{2} \kappa^{2} \rho}\right] C_{0},
\end{aligned}
$$

where $f^{\prime}=d f / d N$ and

$$
a^{2} \kappa^{2} \rho=\beta \exp -\int(1+3 w) d N, \beta=a_{0}^{2} \kappa^{2} \rho_{0} .
$$

Here $\beta$ is dimensionless and can be given an arbitrary positive value by suitable choice of $a_{0}$.

For comparison, in the general relativity case $\left(\rho / \lambda=0=\rho^{*}=U\right)$ these two equations reduce to the single equation

$$
\Phi_{\mathrm{gr}}^{\prime}=-\frac{1}{2}(5+3 w) \Phi_{\mathrm{gr}}+\frac{3}{4}(1+w) C_{0} .
$$

If $w$ is constant, then the non-decaying attractor solution is

$$
\Phi_{\mathrm{gr}}=\frac{3(1+w)}{2(5+3 w)} C_{0} .
$$

Deviations from general relativity arise from early-universe high-energy effects (when $\rho \gg \lambda$ ), and from the KK effects encoded in $\rho^{*}$ and $U$. At late times, the high-energy corrections become negligible. The background dark radiation decays like ordinary radiation $\left(\rho^{*} \propto a^{-4} \propto \rho_{\mathrm{rad}}\right)$, and is negligible at late times by Eq. (5). However, it can leave a signature that survives at late times, as we show below.

\section{A. No dark radiation in the background}

Equation (31) is much simpler in the case when there is no dark radiation in the background $\left(\rho^{*}=0\right)$, i.e. when there is no bulk black hole. (Note also that the errors in [12] do not affect this case, so that the solutions given there are correct.) When $\rho^{*}=0$ we get

$$
U=U_{0} \exp -\int(1-3 w) d N
$$

Since $\rho^{*}=0$, it follows from Eq. (18) that $U$ is an isocurvature mode. This isocurvature mode associated with dark radiation perturbations therefore decays for all equations of state less stiff than radiation, i.e. for $w<\frac{1}{3}$.

If $w$ is constant, then Eq. (30) becomes

$$
\Phi^{\prime}=-\left[\frac{(5+3 w)+(4+3 w) \rho / \lambda}{2+\rho / \lambda}\right] \Phi-\left[\frac{3(1+w)}{2(1+\rho / 2 \lambda)}\right] e^{-2 N} \tilde{U}_{0}+\left[\frac{3(1+w)}{4(1+\rho / 2 \lambda)}\right] C_{0},
$$

where $\tilde{U}_{0}=\beta U_{0}$. The $U$ contribution to $\Phi$ is suppressed at low energies, even if $w>\frac{1}{3}$ (when $U$ grows). Thus the general relativity solution Eq. (34) for constant $w$ is a low-energy attractor for $\rho^{*}=0$ :

$$
\Phi_{\text {low }} \approx \frac{3(1+w)}{2(5+3 w)} C_{0} .
$$

Thus there is no surviving imprint in $\Phi$ of the dark radiation perturbations (the isocurvature mode) when $\rho^{*}=0$ in the background.

At high energies/ early times, Eq. (36) becomes 


$$
\Phi^{\prime} \approx-(4+3 w) \Phi-\frac{\lambda}{\rho_{0}}[3(1+w)] e^{(1+3 w) N} \tilde{U}_{0}+\frac{\lambda}{\rho_{0}}\left[\frac{3}{2}(1+w)\right] e^{3(1+w) N} C_{0},
$$

where $\lambda \ll \rho_{0}$. Thus in the early universe, the contribution of the entropy mode $U$ is sensitive to the equation of state. This contribution decays for $w<-\frac{1}{3}$, including the case of inflation. For $w \geq-\frac{1}{3}$, Eq. (38) shows that the non-decaying solution is

$$
\Phi_{\text {high }} \approx \frac{\lambda}{\rho_{0}}\left[\frac{3(1+w)}{2(7+6 w)} C_{0}\right] e^{3(1+w) N}-\frac{\lambda}{\rho_{0}}\left[\frac{3 \beta(1+w)}{(5+6 w)} U_{0}\right] e^{(1+3 w) N},
$$

so that in the very high energy limit,

$$
\Phi_{\text {high }} \rightarrow \frac{3}{2} \frac{\lambda}{\rho_{0}}(1+w)\left[\frac{C_{0}}{7+6 w}-\frac{2 \tilde{U}_{0}}{5+6 w}\right]
$$

Hence, for constant $w\left(\geq-\frac{1}{3}\right)$, high-energy effects initially suppress $\Phi$ (by the factor $\left.\lambda / \rho_{0}\right)$, before it starts to grow. This growth leads to the late-time general relativity value, Eq. (37). By contrast, in general relativity, $\Phi$ remains constant when $w$ is constant.

The most relevant case is $w=\frac{1}{3}$. If inflation takes place on the brane, reheating will initiate a radiation era. Provided the reheat temperature is high enough, the radiation era will start at high energies. Large-scale perturbations $\Phi$ will be amplified according to Eqs. (40) and (37) with $w=\frac{1}{3}$.

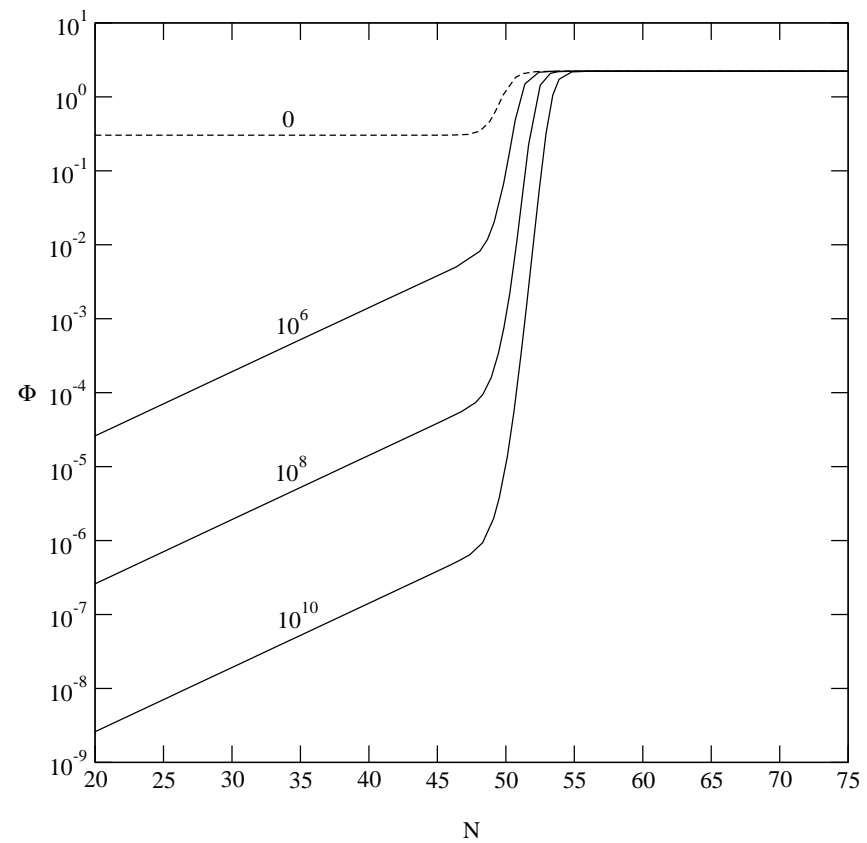

FIG. 1. The evolution of $\Phi$ for a mode that is well beyond the Hubble radius at $N=0$, about 50 e-folds before inflation ends, and remains super-Hubble through the radiation era. A smooth transition from inflation to radiation is modelled by $w=\frac{1}{2}\left[\left(\frac{4}{3}-\epsilon\right) \tanh (N-50)-\left(\frac{2}{3}-\epsilon\right)\right]$, where $\epsilon$ is a small positive parameter, chosen as $\epsilon=1 / 15$ in the plot. (The parameter $\beta$ in Eq. (32) is set to 1 ). Labels on the curves give the value of $\rho_{0} / \lambda$; the general relativity solution is the dashed curve.

For slow-roll inflation, $w$ is not constant, but close to -1 and slowly increasing, i.e., $1+w \sim \epsilon$, with $0<\epsilon \ll 1$ and $H^{-1}|\dot{\epsilon}|=\left|\epsilon^{\prime}\right| \ll 1$. Then Eq. (38) leads to

$$
\Phi_{\text {high }} \sim \frac{3}{2} \epsilon \frac{\lambda}{\rho_{0}} C_{0} e^{3 \epsilon N},
$$

so that $\Phi$ has a slowly growing mode during high-energy slow-roll inflation. This is different from general relativity, where $\Phi$ is constant during slow-roll inflation. Thus more amplification of $\Phi$ can be achieved than in general relativity, consistent with Eq. (8). The evolution of $\Phi$ is illustrated for a toy model of inflation-to-radiation in Fig. 1 (from [12]). The early (growing) and late time (constant) attractor solutions are seen explicitly in the plots. 


\section{B. Nonzero dark radiation in the background}

The presence of dark radiation in the background has interesting qualitative implications, even though the quantitative constraints from observations on the amount of dark radiation are quite severe. The general relativity solution, Eq. (34) is no longer the late-time attractor when $\rho^{*} \neq 0$.

The dark radiation factor, for constant $w$, behaves as

$$
\frac{\rho^{*}}{\rho}=\alpha e^{(3 w-1) N}, \quad \alpha=\frac{\rho_{0}^{*}}{\rho_{0}},
$$

where $\alpha$ is a dimensionless constant. For equations of state less stiff than radiation $\left(w<\frac{1}{3}\right)$, the dark radiation factor redshifts away. If $w>\frac{1}{3}$, then this factor grows with expansion. In the radiation era, $w=\frac{1}{3}$, the dark radiation factor is constant.

If there was a period of inflation on the brane before the radiation era, then dark radiation would be redshifted to negligible levels. However, the reheating era that creates the radiation, could at the same time create dark radiation via $5 \mathrm{D}$ graviton emission in high-energy interactions [20]. The fraction of dark radiation is limited by observational constraints, Eq. (5), so that

$$
\alpha \lesssim 0.03
$$

We can therefore perform a perturbative solution of Eqs. (30) and (31), up to first order in $\alpha$. The zero-order solutions are given in the previous subsection.

We find that the non-decaying solutions for radiation in the low-energy regime are

$$
\begin{aligned}
& \Phi_{\text {low }} \approx \frac{C_{0}}{3}(1-\alpha), \\
& \tilde{U}_{\text {low }} \approx \tilde{U}_{0}+\alpha \frac{C_{0}}{3} e^{2 N} .
\end{aligned}
$$

It follows that the first-order correction to $\tilde{U}$ (where $\tilde{U}=\tilde{U}^{(0)}+\tilde{U}^{(1)}$ ) behaves like the large-scale density perturbations for radiation in general relativity:

$$
\tilde{U}_{\text {low }}^{(1)} \propto \Delta \propto a^{2}
$$

In the high-energy regime, the non-decaying solutions are

$$
\begin{aligned}
& \Phi_{\text {high }} \approx \frac{\lambda}{\rho_{0}}\left[\frac{2}{9} C_{0} e^{4 N}-\frac{4}{7} \tilde{U}_{0} e^{2 N}\right]+4 \alpha\left(\frac{\lambda}{\rho_{0}}\right)^{2}\left[\left(\frac{2}{63} C_{0}-\frac{4}{49} \tilde{U}_{0}\right) e^{2 N}+\frac{4}{77} \tilde{U}_{0} e^{6 N}-\frac{2}{117} C_{0} e^{8 N}\right] \\
& \tilde{U}_{\text {high }} \approx \tilde{U}_{0}+\alpha \frac{\lambda}{\rho_{0}}\left[\frac{2}{9} C_{0}\left(e^{6 N}-1\right)-\frac{4}{7} \tilde{U}_{0}\left(e^{4 N}-1\right)\right] .
\end{aligned}
$$

In the very high energy limit,

$$
\begin{aligned}
& \Phi_{\text {high }} \rightarrow \frac{\lambda}{\rho_{0}}\left[\frac{2}{9} C_{0}-\frac{4}{7} \tilde{U}_{0}\right]+16 \alpha\left(\frac{\lambda}{\rho_{0}}\right)^{2}\left[\frac{C_{0}}{273}-\frac{4 \tilde{U}_{0}}{539}\right], \\
& \tilde{U}_{\text {high }} \rightarrow \tilde{U}_{0} .
\end{aligned}
$$

Comparing Eq. (49) with Eq. (40) shows that the high energy limit with dark radiation differs only negligibly from the no-dark-radiation limit. 


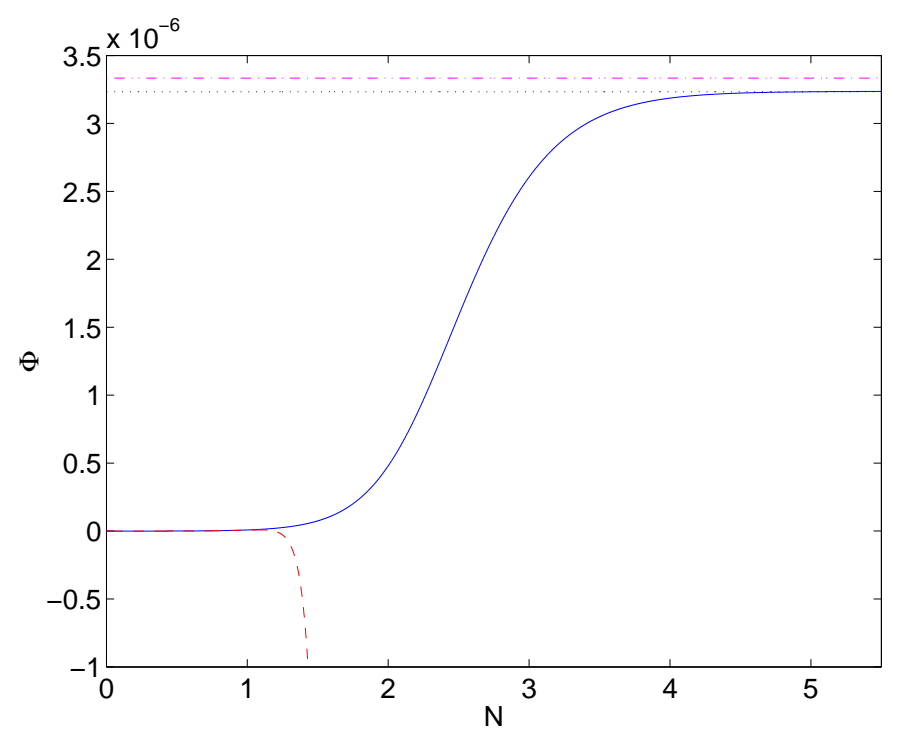

FIG. 2. The evolution of $\Phi$ on very large scales in the radiation era, with $\rho_{0} / \lambda=10^{4}$ and dark radiation factor $\alpha=0.03$. Initial conditions are given by $C_{0}=10^{-5}=\tilde{U}_{0}$, and $\Phi$ is initially set to its high-energy attractor, Eq. (49). The solid curve is the numerical integration. The dashed curve is the high-energy approximation, Eq. (47). The dotted curve is the low-energy approximation, Eq. (44), and the dot-dashed curve is the low-energy approximation without dark radiation $(\alpha=0)$.

This analysis shows that $\Phi$ is initially suppressed, then begins to grow, as in the no-dark-radiation case, eventually reaching an attractor which is less than the no-dark-radiation attractor. The role of dark radiation is to suppress, by the small fractional amount $\alpha$, the final value of $\Phi$, given in Eq. (44). The dark radiation perturbation $U$ starts from a constant value at very high energies, and then grows at late times like the density perturbations in radiation. Figures 2 and 3 confirm the qualitative analysis.

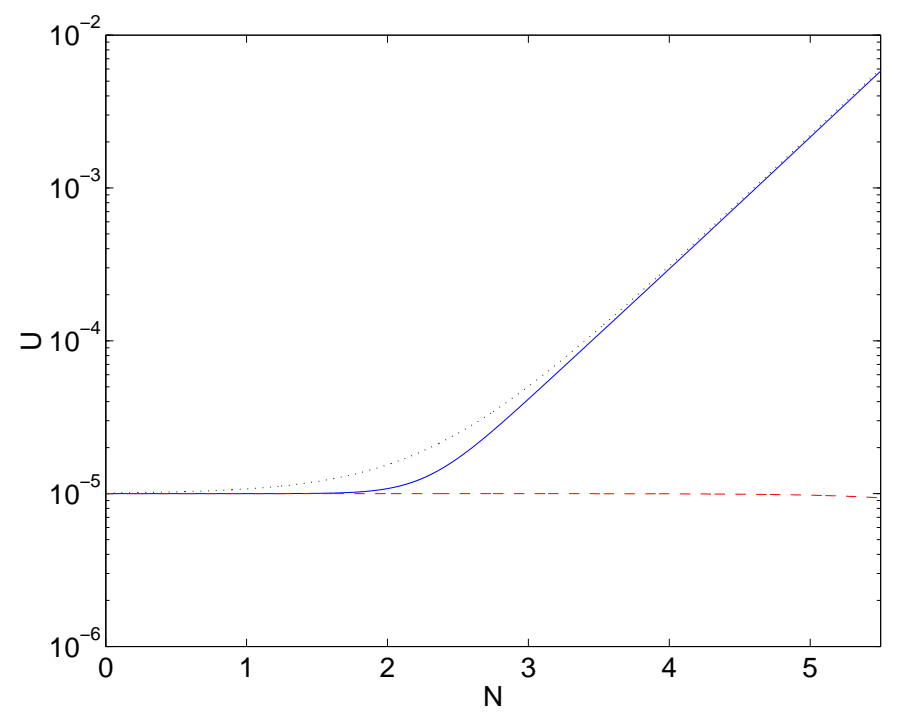

FIG. 3. The evolution of $U$, with the same conditions as in Fig. 2. The dashed curve is the high-energy approximation, Eq. (48), and the dotted curve is the low-energy approximation, Eq. (45).

The effective total entropy, Eq. (18), has the following behaviour, to $O(\alpha)$, in the radiation era:

$$
\begin{aligned}
& S_{\text {high }}^{\mathrm{eff}} \rightarrow-\frac{8}{5} \frac{\lambda}{\rho_{0}}\left[U_{0}-\alpha \frac{\lambda}{\rho_{0}}\left(\frac{2 C_{0}}{9 \beta}-\frac{4}{7} U_{0}\right)\right], \\
& S_{\mathrm{low}}^{\mathrm{eff}} \approx-4 \frac{\rho_{0}}{\lambda} e^{-4 N}(1-2 \alpha) U_{0} .
\end{aligned}
$$


At high energies $\left|S^{\text {eff }}\right|$ is suppressed by the factor $\lambda / \rho_{0}$, and the $\alpha$-correction is negligible. At low energies, the large factor $\rho_{0} / \lambda$ is overwhelmed by the redshift factor $e^{-4 N}$.

Without dark radiation in the background, $U$ in the radiation era remains constant. When there is dark radiation in the background, $U$ grows. The growth of $U$ leads to a decrease in the final value of $\Phi$.

An extreme case of loss of power in $\Phi$ occurs when the dark radiation redshifts more slowly than the matter, so that it eventually dominates over the matter. This happens when the matter equation of state is stiffer than radiation, $w>\frac{1}{3}$. For example, for stiff matter, $w=1$, we have

$$
\frac{\rho^{*}}{\rho} \propto a^{2}
$$

by Eq. (42). As a result, the decrease in $\Phi$ becomes so pronounced that $\Phi$ is driven to 0 at late times. By Eqs. (30) and (31), we find that

$$
U_{\text {low }} \propto e^{4 N}, \quad \Phi_{\text {low }} \propto e^{-2 N}
$$

at late times. The suppression of $\Phi$ is confirmed by numerical integration, as shown in Fig. 4 .

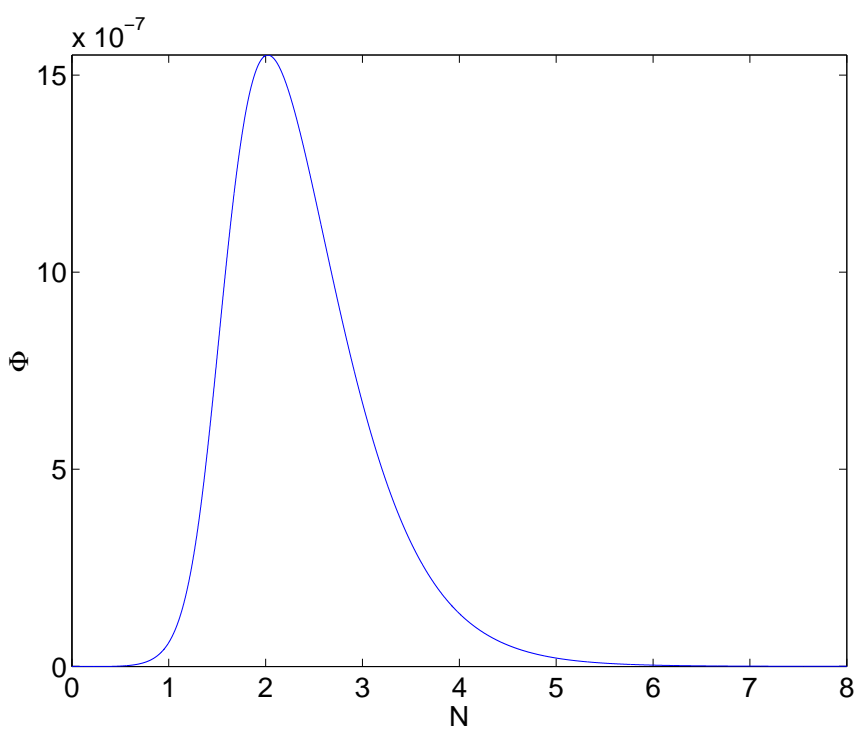

FIG. 4. The evolution of $\Phi$ on large scales in a stiff (kinetic) era (with parameters and initial conditions as in Figs. 2 and 3 ).

This example could be relevant to the steep inflation scenario on the braneworld. High-energy effects mean that steep potentials can drive inflation on the brane when they would not give inflation in general relativity. Typically, this inflation ends in a kinetic regime with $w \approx 1$, and with particle production being gravitational [10]. If dark radiation can be produced by high-energy interactions, then its presence serves to suppress $\Phi$ on large-scales, as in Fig. 4.

\section{CONCLUSION}

We have investigated the effects of dark radiation in the background on large-scale density perturbations (neglecting gradient terms) in a Randall-Sundrum 2 type brane-world. Dark radiation significantly complicates the perturbation equations. Since it is limited to be a small fraction of the radiation energy density, its effects can be qualitatively analysed via a perturbative approach. This approach, confirmed by our numerical simulations, shows that in the radiation era, the large-scale density perturbations are suppressed at late times by a small amount. At the same time, the large-scale perturbations in the dark radiation itself grow at late times. By contrast these perturbations have constant amplitude in a universe with no dark radiation in the background. The suppression of density perturbations becomes strong when the matter has a stiff (kinetic-dominated) equation of state. 


\section{Acknowledgments}

We thank Peter Dunsby for pointing out the minor errors in [12], which are corrected in Eqs. (20)-(22). BG is supported by the British-Thai Scholarship Scheme and a Royal Thai Government Scholarship. CG and RM are supported by PPARC.

[1] P. Binetruy, C. Deffayet, U. Ellwanger and D. Langlois, Phys. Lett. B477, 285 (2000).

[2] P. Kraus, JHEP 12, 011 (1999);

S. Mukohyama, Phys. Lett. B473, 241 (2000);

D. Ida, JHEP 09, 014 (2000);

S. Mukohyama, T. Shiromizu and K. Maeda, Phys. Rev. D 61, 024028 (2000);

E.E. Flanagan, S.-H. Henry Tye and I. Wasserman, Phys. Rev. D 62, 044039 (2000);

P. Bowcock, C. Charmousis and R. Gregory, Class. Quantum Grav. 17, 4745 (2000).

[3] T. Shiromizu, K. Maeda and M. Sasaki, Phys. Rev. D 62, 024012 (2000).

[4] R. Maartens, D. Wands, B.A. Bassett and I.P.C. Heard, Phys. Rev. D 62, 041301 (2000).

[5] L. Randall and R. Sundrum, Phys. Rev. Lett. 83, 4690 (1999).

[6] J. Garriga and T. Tanaka, Phys. Rev. Lett. 84, 2778 (2000).

[7] J.D. Barrow and R. Maartens, Phys. Lett. B532, 153 (2002).

[8] D. Langlois, R. Maartens, M. Sasaki and D. Wands, Phys. Rev. D 63, 084009 (2001).

[9] K. Ichiki, M. Yahiro, T. Kajino, M. Orito and G.J. Mathews, Phys. Rev. D 66, 043521 (2002); J.D. Bratt, A.C. Gault, R.J. Scherrer and T.P. Walker, Phys. Lett. B546, 19 (2002).

[10] E.J. Copeland, A.R. Liddle and J.E. Lidsey, Phys. Rev. D 64, 023509 (2001);

A. S. Majumdar, Phys. Rev. D 64, 083503 (2001);

G. Huey and J.E. Lidsey, Phys. Lett. B514, 217 (2001);

V. Sahni, M. Sami and T. Souradeep, Phys. Rev. D 65, 023518 (2002);

N.J. Nunes and E.J. Copeland, Phys. Rev. D 66, 043524 (2002);

A.R. Liddle, L.A. Urena-Lopez, astro-ph/0302054.

[11] R. Maartens, Phys. Rev. D 62, 084023 (2000).

[12] C. Gordon and R. Maartens, Phys. Rev. D 63, 044022 (2001).

[13] S. Mukohyama, Phys. Rev. D 62, 084015 (2000);

S. Mukohyama, Phys. Rev. D 64, 064006 (2001).

[14] R. Maartens, gr-qc/0101059.

[15] B. Leong, P.K.S. Dunsby, A.D. Challinor and A.N. Lasenby, Phys. Rev. D 65, 104012 (2002).

[16] M. Bruni and P.K.S. Dunsby, Phys. Rev. D 66, 101301 (2002).

[17] B. Leong, A.D. Challinor, R. Maartens and A.N. Lasenby, Phys. Rev. D 66, 104010 (2002).

[18] M. Bruni, P.K.S. Dunsby and G.F.R. Ellis, Astrophys. J. 395, 34 (1992).

[19] K. Koyama, astro-ph/0303108.

[20] D. Langlois, L. Sorbo and M. Rodriguez-Martinez, Phys. Rev. Lett. 89, 171301 (2002). 